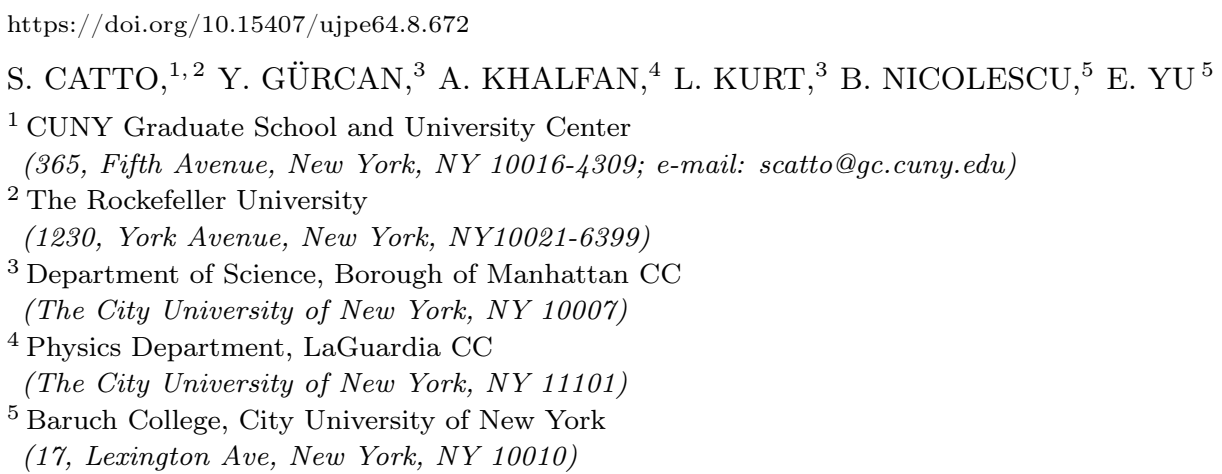

\title{
HADRONIC SUPERSYMMETRY FROM QCD
}

The evolution of hadronic mass formulae with special emphasis on group theoretical descriptions and supersymmetry suggested by QCD and based on quark-antidiquark symmetry is shown, with further comments on possible applications to a Skyrme-type models that may compete with the potential quark models in the future.

Ke ywords: supersymmetry, quark models, skyrmions.

\section{Introduction}

The quark model with potentials derived from QCD, including the quark-diquark model for excited hadrons gives mass formulae in a very good agreement with experiments and goes a long way in explaining the approximate symmetries and supersymmetries of the hadronic spectrum, including the symmetry breaking mechanism.

The mathematical expression of supersymmetry arises through a generalization of Lie algebras to superalgebras. When a Lie algebra is $s u(n)$ it can be extended to a graded algebra (superalgebra) $s u(n / m)$ with even and odd generators, the even generators being paired with commuting (bosonic) parameters and the odd generator with the Grassmann (fermionic) parameters. The algebra can then be exponentiated to the supergroup $\mathrm{SU}(n / m)$. This was done by Miyazawa [1] who derived the correct commutation and anticommutation relations for such a superalgebra, as well as the generalized Jacobi identity. This discovery predates the supersymmetry in dual resonance models or supersymmetry in quantum field theories invariant under the super-Poincaré group that generalizes special relativity. Miyazawa looked for a supergroup that would contain SU(6)

(c) S. CATTO, Y. GÜRCAN, A. KHALFAN, L. KURT, B. NICOLESCU, E. YU, 2019 and settled on broken $\mathrm{SU}(6 / 21)$. He showed that an $\mathrm{SU}(3)$ singlet-octet of this supergroup leads to a new kind of mass formulae relating fermionic and bosonic mass splittings.

\section{Quark-Diquark Model}

We shall first discuss the validity domain of $\mathrm{SU}(6 / 21)$ supersymmetry $[2,3,6]$. The diquark structure with spins $s=0$ and $s=1$ emerges in inelastic inclusive lepton-baryon collisions with high impact parameters that excite the baryon rotationally, resulting in inelastic structure functions based on point-like quarks and diquarks instead of three point-like quarks. In this case, both mesons and baryons are bilocal with large separation of constituents.

In addition, there is a symmetry between color antitriplet diquarks with $s=0$ and $s=1$ and color antitriplet antiquarks with $s=\frac{1}{2}$. This is only possible, if the force between quark $q$ and antiquark $\bar{q}$, and between $q$ and diquark $D$ is mediated by a zero spin object that sees no difference between the spins of $\bar{q}$ and $D$. The object can be in color states that are either singlet or octet since $q$ and $D$ are both triplets. Such an object is provided by scalar flux tubes of gluons that dominate over the one gluon exchange at large distances. Various strong coupling approximations to QCD, like lattice gauge theory $[4,5]$, 't Hooft's $\frac{1}{N}$ approximation [7], when $N$, the number of colors, is

ISSN 2071-0194. Ukr. J. Phys. 2019. Vol. 64, No. 8 
very large, or the elongated bag model [8] all give a linear potential between widely separated quarks and an effective string that approximates the gluon flux tube. In such a theory, it is energetically favorable for the three quarks in a baryon to form a linear structure with a quark in the middle and two at the ends or, for a high rotational excitation, a bilocal linear structure (diquark) at one end and a quark at the other end. In order to illustrate these points, we start with the suggestion of Johnson and Thorn [8] that the string-like hadrons may be pictured as the vortices of color flux lines which terminate on the concentration of color at the end points. The color flux connecting opposite ends is the same for mesons and baryons giving an explanation for the same slope of meson and baryon trajectories [3].

To construct a solution, which yields a maximal angular momentum for a fixed mass, we consider a bag with elongated shape rotating about the center of mass with an angular frequency $\omega$. Its ends have the maximal velocity allowed, which is the speed of light $(c=1)$. Thus, a given point inside the bag, at a distance $r$ from the axis of rotation moves with a velocity

$v=|\boldsymbol{\omega} \times \mathbf{r}|=\frac{2 r}{L}$,

where $L$ is the length of the string. In this picture, the bag surface will be fixed by balancing the gluon field pressure against the confining vacuum pressure $B$, which (in analogy to electrodynamics) can be written in the form

$\frac{1}{2} \sum_{\alpha=1}^{8}\left(E_{\alpha}^{2}-B_{\alpha}^{2}\right)=B$

Using Gauss' law, the color electric field $E$ through the flux tube connecting the color charges at the ends of the string is given by

$\int \mathbf{E}_{\alpha} d \mathbf{S}=E_{\alpha} A=g \frac{1}{2} \lambda_{\alpha}$,

where $A(r)$ is the cross-section of the flux tube at distance $r$ from the center and $g \frac{1}{2} \lambda_{\alpha}$ is the color electric charge, which is the source of $E_{\alpha}$. By analogy with classical electrodynamics, the color magnetic field $\mathbf{B}_{\alpha}(r)$ associated with the rotation of the color electric field is

$\mathbf{B}_{\alpha}(r)=\mathbf{v}(r) \times \mathbf{E}_{\alpha}(r)$, at a point moving with a velocity $\mathbf{v}(r)$. For the absolute values, this yields

$B_{\alpha}=v E_{\alpha}$,

because $\mathbf{v}(r)$ is perpendicular to $\mathbf{E}_{\alpha}(r)$. Using last three equations together with

$\left\langle\sum_{\alpha=1}^{8}\left(\frac{1}{2} \lambda_{\alpha}\right)^{2}\right\rangle=\frac{4}{3}$

for the $S U(3)^{c}$ triplet in Eq. (2), we obtain that the cross-section of the bag

$A(r)=\sqrt{\frac{2}{3 B}} g \sqrt{1-v^{2}}$,

which shows the expected Lorentz contraction.

The total energy $E$ of the bag

$E=E_{q}+E_{G}+B V$

is the sum of the quark energy $E_{q}$, the gluon field energy $E_{G}$, and the volume energy of the bag, $B V$. Because the quarks at the ends move with the a speed close to the speed of light, their energy is simply given by

$E_{q}=2 p$,

where $p$ is the momentum of a quark, a diquark, or an antiquark, respectively. By analogy with electrodynamics, Eqs. (3)-(5) yield

$E_{G}=\frac{1}{2} \int d^{3} x \sum_{\alpha=1}^{8}\left(E_{\alpha}^{2}+B_{\alpha}^{2}\right)=$

$=\sqrt{\frac{2}{3}} g \sqrt{B} L \int_{0}^{1} d v \frac{1+v^{2}}{\sqrt{1-v^{2}}}=\sqrt{\frac{2}{3}} g \sqrt{B} L \frac{3 \pi}{4}$

for the gluon energy and

$B V=2 B \int_{0}^{\frac{L}{2}} A(r) d r=$

$=2 B \int_{0}^{1} \sqrt{\frac{2}{3 B}} g \sqrt{1-v^{2}} \frac{L}{2} d v=$

$=\sqrt{\frac{2}{3}} g \sqrt{B} L \frac{\pi}{4}=\frac{B A(0) L \pi}{4}$

ISSN 2071-0194. Ukr. J. Phys. 2019. Vol. 64, No. 8 
for the volume energy. It is obvious from Eq. (10) that the gluon field energy is proportional to the length $L$ of the bag. The gluon field energy and the volume energy of the bag together correspond to a linear rising potential of the form

$V(L)=E_{G}+B V=b L$,

where

$b=\sqrt{\frac{2 B}{3}} g \pi$.

The total angular momentum $J$ of this classical bag is the sum of the angular momenta of the quarks at the two ends

$J_{q}=p L$

and the angular momentum $J_{G}$ of the gluon field. From Eq. (4), we get

$\mathbf{E}_{\alpha} \times \mathbf{B}_{\alpha}=\mathbf{v} E_{\alpha}^{2}$

for the momentum of the gluon field. Hence,

$J_{G}=\left|\int_{\text {bag }} d^{3} \mathbf{r} \sum_{\alpha=1}^{8} \mathbf{r} \times\left(\mathbf{E}_{\alpha} \times \mathbf{B}_{\alpha}\right)\right|=$
$=2 \int_{0}^{\frac{L}{2}} d r A(r) r v E_{\alpha}^{2}=\frac{16}{3 L} g^{2} \int_{0}^{\frac{L}{2}} \frac{r^{2} d r}{A(r)}=\sqrt{\frac{2}{3}} g \sqrt{B} L^{2} \frac{\pi}{4}$,

where we have used Eq. (1) and Eq. (3) in the third step. We can now express the total energy of the bag in terms of angular momenta. Putting these results back into the formulae for $E_{q}$ and $E_{G}$, we arrive at

$E_{q}=\frac{2 J_{q}}{L}, \quad E_{G}=\frac{3 J_{G}}{L}$,

so that the bag energy now becomes

$$
\begin{aligned}
& E=\frac{2 J_{q}}{L}+\frac{3 J_{G}}{L}+\sqrt{\frac{2 B}{3}} L g \frac{\pi}{L}= \\
& =\frac{2 J_{q}+4 J_{G}}{L}=\frac{2\left(J+J_{G}\right)}{L}= \\
& =\frac{1}{L}\left(2 J+\sqrt{\frac{2}{3}} g \sqrt{B} L^{2} \frac{\pi}{2}\right) .
\end{aligned}
$$

Minimizing the total energy for a fixed angular momentum with respect to the length of the bag, $\frac{\partial E}{\partial L}=0$ gives the relation

$$
-\frac{2 J}{L^{2}}+\sqrt{\frac{2}{3}} g \sqrt{B} \frac{\pi}{2}=0
$$

so that

$L^{2}=\frac{4 J}{g \pi} \sqrt{\frac{3}{2 B}}$.

Re-inserting this into Eq. (18), we arrive at

$E=2 \sqrt{J g \pi}\left(\frac{2 B}{3}\right)^{\frac{1}{4}}$

or

$J=\left(\sqrt{\frac{3}{2 B}} \frac{1}{4 \pi g}\right) E^{2}=$

$=\left(\sqrt{\frac{3}{2 B}} \frac{1}{8 \pi^{\frac{3}{2}}} \frac{1}{\sqrt{\alpha_{s}}}\right) E^{2}=\alpha^{\prime}(0) M^{2}$,

where $M=E$, and $\alpha_{s}=\frac{g^{2}}{4 \pi}$ is the unrationalized color gluon coupling constant. We can now let $\alpha^{\prime}(0)$ defined by the last equation, which is the slope of the Regge trajectory, be expressed as

$\alpha^{\prime}(0)=\sqrt{\frac{3}{2 B}} \frac{1}{8 \pi^{\frac{3}{2}}} \frac{1}{\sqrt{\alpha_{s}}}=\frac{1}{4 b}$,

where $b$ was defined in Eq. (12).

The parameters $B$ and $\alpha_{s}$ have been determined $[9,10]$ using the experimental information from the low lying hadron states: $B^{\frac{1}{4}}=0.146 \mathrm{GeV}$ and $\alpha_{s}=$ $=0.55 \mathrm{GeV}$. If we use these values in Eq. (23), we find

$\alpha^{\prime}(0)=0.88(\mathrm{GeV})^{-2}$

in the remarkable agreement with the slope determined from experimental data, which is about $0.9(\mathrm{GeV})^{-2}$.

Then the total phenomenological non-relativistic potential is the well-known superposition of the Coulomb-like and confining potentials $V(r)=\frac{a}{r}+b r$, where $r=\left|\mathbf{r}_{1}-\mathbf{r}_{2}\right|$ is the distance between $q$ and $\bar{q}$ in a meson or between $q$ and $D$ in a baryon with high angular momentum. This was verified in lattice QCD to a high degree of accuracy [11] $\left(a=\frac{-c \alpha_{c}}{r}\right.$, where

ISSN 2071-0194. Ukr. J. Phys. 2019. Vol. 64, No. 8 
$c$ is the color factor, and $\alpha_{c}$ is the strong coupling strength).

It is interesting to know that all this is related very closely to the dual strings. Indeed, we can show that the slope given in Eq. (23) is equivalent to the dual string model formula for the slope, if we associate the "proper tension" in the string with the proper energy per unit length of the color flux tube and the volume. By the proper energy per unit length, we mean the energy per unit length at a point in the bag evaluated in the rest system of that point. This will be

$T_{0}=\frac{1}{2} \sum_{\alpha} E_{\alpha}^{2} A_{0}+B A_{0}$

The relation $\frac{1}{2} \sum_{\alpha} E_{\alpha}^{2}=B$ in the rest system gives

$T_{0}=2 B A_{0}$,

where $A_{0}$ is the cross-sectional area of the bag. Let $A=A_{0}$ in Eq. (7), when $v=0$. Then, using

$A_{0}=\sqrt{\frac{2}{3 B}} g$,

we find

$T_{0}=2 \sqrt{\frac{2}{3}} g \sqrt{B}=4 \sqrt{\frac{2 \pi}{3}} \sqrt{\alpha_{s}} \sqrt{B}$

for the proper tension. In the dual string, the slope and the proper tension are related by the formula [12]

$T_{0}=\frac{1}{2 \pi \alpha^{\prime}}$,

so that the slope is

$\alpha^{\prime}=\frac{1}{8} \sqrt{\frac{3}{2}} \frac{1}{\pi^{\frac{3}{2}}} \frac{1}{\sqrt{\alpha_{s}}} \frac{1}{\sqrt{B}}$,

which is identical to the earlier formula we produced in Eq. (23).

It would appear from Eq. (28) that the ratio of volume to field energy would be one-to-one in one space dimension in contrast to the result one-to-three, which holds for a three-dimensional bag [13]. However, the ratio one-to-one is true only in the rest system at a point in the bag, and each position along the $x$ axis is, of course, moving with a different velocity. Indeed, we see from Eq. (10) and Eq. (11) that the ratio of the total volume energy to the total field energy is given by one-to-three in conformity with the virial theorem [13].

In the string model of hadrons, we have $E^{2} \sim J$ between the energy and the angular momentum of the rotating string. If we denote, by $\rho(r)$, the mass density of a string, and, by $v$ and $\omega$, its linear and angular velocities, respectively, the energy and the angular momentum of the rotating string are given by

$E=2 \int \frac{\rho(r)}{\sqrt{1-\omega^{2} r^{2}}} d r=\frac{2}{\omega} \int_{0}^{1} \frac{\rho(v)}{\sqrt{1-v^{2}}} d v$

and

$J=2 \int \frac{\rho(r)}{\sqrt{1-\omega^{2} r^{2}}} r^{2} \omega d r=\frac{2}{\omega^{2}} \int_{0}^{1} \frac{\rho(v)}{\sqrt{1-v^{2}}} v^{2} d v$.

Hence, the relation

$E^{2} \propto J$

holds. If the string is loaded with mass points at its ends, they no longer move with the speed of light. However, the above relation still holds approximately for the total energy and angular momentum of the loaded string.

We now look at various ways of the partitioning of the total angular momentum into two subsystems. Figures $a, b$, and $c$ show the possible configurations of three quarks in a baryon. If we put the proportionality constant in Eq. (33) equal to unity, then the naive evaluation of energies yield

$E^{2}=J_{1}+J_{2}=E_{1}^{2}+E_{2}^{2} \leq\left(E_{1}+E_{2}\right)^{2}=E^{\prime 2}$,

where $E$ and $E^{\prime}$ denote the energies corresponding to Figures $a$ or $c$. In the case of Figure $b, J_{1}$ and $J_{2}$ are the angular momenta corresponding to the energies $E_{1}$ and $E_{2}$ of the subsystems. The equality in Eq. (34) holds, only if $E_{1}$ or $E_{2}$ is zero. Therefore, for each fixed total angular momentum, its most unfair partition into two subsystems gives us the lowest energy levels, and its more or less fair partition gives rise to energy levels on daughter trajectories. Hence, on the leading baryonic trajectory, we have a quarkdiquark structure (Fig. a) or a linear molecule structure (Fig. c). On the other hand, on low-lying trajectories, we have more or less symmetric $\left(J_{1} \sim J_{2}\right)$ configuration of quarks. 


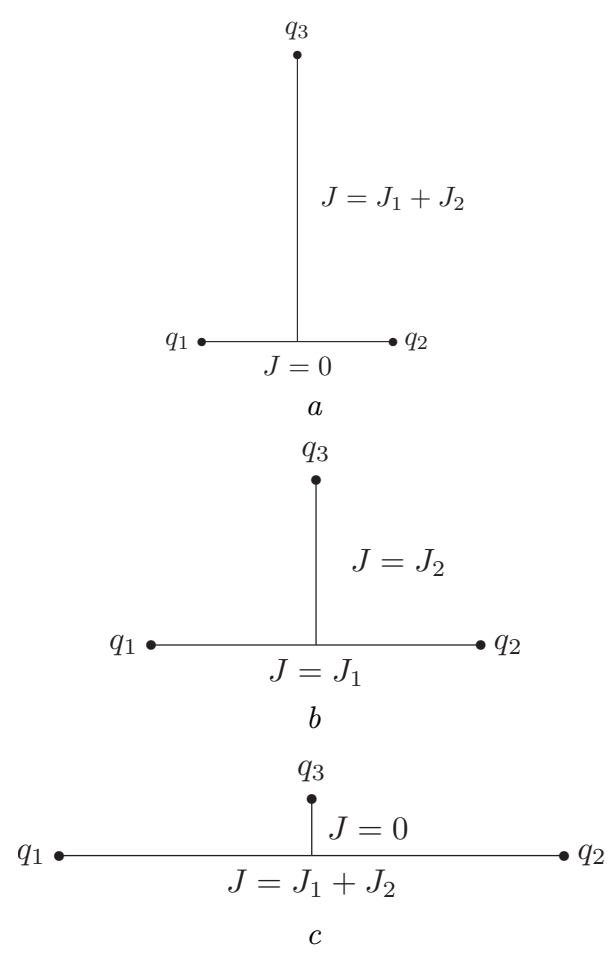

Since the high- $J$ hadronic states on leading Regge trajectories tend to be bilocal with large separation of their constituents, they fulfill all the conditions for supersymmetry between $\bar{q}$ and $D$. Then the only difference between the energies of $(q \bar{q})$ mesons and $(q D)$ baryons comes from the different masses of their constituents, namely, $m_{q}=m_{\bar{q}}=m$, and $m_{D} \sim 2 m$. For high $J$, this is the main source of symmetry breaking, which is spin-independent. We will show how we can obtain sum rules from this breaking. The part of the mass operator that gives rise to this splitting is a diagonal element of $\mathrm{U}(6 / 21)$ that commutes with $\mathrm{SU}(6)$.

Let us now consider the spin-dependent breaking of $\mathrm{SU}(6 / 21)$. For low $J$ states, the $(q D)$ system becomes trilocal $(q q q)$, and the flux tube degenerates to a single gluon propagator that gives spin-dependent forces in addition to the Coulomb term $\frac{a}{r}$. In this case, we have the regime studied by de Rujula, Georgi, and Glashow, where the breaking is due to the hyperfine splitting caused by the exchange of single gluons that have spin 1 . These mass splittings give rise to different intercepts of the Regge trajectories given by

$\Delta m_{12}=k \frac{\mathbf{S}_{1} \mathbf{S}_{2}}{m_{1} m_{2}}, \quad k=|\psi(0)|^{2}$, both for baryons and mesons at high energies. But, at low energies, the baryon becomes a trilocal object (with three quarks), and the mass splitting is given by

$\Delta m_{123}=\frac{1}{2} k\left(\frac{\mathbf{S}_{\mathbf{1}} \mathbf{S}_{\mathbf{2}}}{m_{1} m_{2}}+\frac{\mathbf{S}_{2} \mathbf{S}_{3}}{m_{2} m_{3}}+\frac{\mathbf{S}_{3} \mathbf{S}_{1}}{m_{3} m_{1}}\right)$,

where $m_{1}, m_{2}$, and $m_{3}$ are the masses of the three different quark constituents.

The element of $\mathrm{SU}(6 / 21)$ that gives rise to such splittings is a diagonal element of its $\mathrm{U}(21)$ subgroup and gives rise to $s(s+1)$ terms that behave like an element of the (405) representation of $\mathrm{SU}(6)$ in the $\mathrm{SU}(6)$ mass formulae. The splitting of isospin multiplets is due to a symmetry breaking element in the (35) representation of $\mathrm{SU}(6)$. Hence, all symmetry breaking terms are in the adjoint representation of $\mathrm{SU}(6 / 21)$. If we restrict ourselves to the non-strange sector of hadrons with approximate $\mathrm{SU}(4)$ symmetry, the effective supersymmetry will relate the splitting in $m^{2}$ between $\Delta\left(s=\frac{3}{2}, I=\frac{3}{2}\right)$, and $N\left(s=\frac{1}{2}, I=\frac{1}{2}\right)$ to the splitting between $\omega(s=1, I=0)$ and $\pi$ $(s=0, I=1)$, so that

$m_{\Delta}^{2}-m_{N}^{2}=m_{\omega}^{2}-m_{\pi}^{2}$,

which is satisfied to within $5 \%$. Our potential model gives a more accurate symmetry breaking

$\frac{9}{8}\left(m_{\Delta}^{2}-m_{N}^{2}\right)=m_{\omega}^{2}-m_{\pi}^{2}$

to within $1 \%$, where the $\frac{8}{9}$ arises from $\frac{1}{2}\left(\frac{4}{3} \alpha_{s}\right)^{2}=$ $=\frac{8}{9} \alpha_{s}^{2}$. For a classification of supergroups including $\mathrm{SU}(m / n)$, we refer to the paper by Viktor Kac [14].

\section{Conclusions and Future Prospects}

Effective Hamiltonians and new mass relations including quark and diquark masses were worked out in our previous works that included the complete understanding of hadronic color algebras as well. In the case of heavy quarks, one can also use the nonrelativistic approximation, so that the potential models for the spectrum of charmonium and the $b \bar{b}$ system can be worked out. In such an approach, gluons can be eliminated leaving quarks interacting through potentials.

It is also possible to take an opposite approach by eliminating quarks as well as gluons, leaving only an effective theory that involves mesons and baryons as

ISSN 2071-0194. Ukr. J. Phys. 2019. Vol. 64, No. 8 
collective excitations (solitons) in a way by Skyrme. A Skyrme model that can compete with the potential model is not yet realized.

It is a pleasure to acknowledge helpful conversations with Professors Vladimir Akulov, Cestmir Burdik, and Francesco Iachello.

The work is supported in part by DOE contracts Nos. DE-AC-0276-ER 03074 and 03075; NSF Grant No. DMS-8917754; and several PSC-CUNY research grants.

1. H. Miyazawa. Baryon number changing currents. Prog. Theor. Phys. 36, 1266 (1966); Spinor currents and symmetries of baryons and mesons. Phys. Rev. 170, 1586 (1968).

2. S. Catto, F. Gürsey. New realizations of hadronic supersymmetry. Nuovo Cimento A 99, 685 (1988).

3. S. Catto, F. Gürsey. Algebraic treatment of effective supersymmetry. Nuovo Cimento A 86, 201 (1985).

4. K.N. Wilson. Confinement of quarks. Phys. Rev. D 10, 2445 (1974)

5. R.L. Jaffe. Multiquark hadrons. II. Methods. Phys. Rev. D 15, 281 (1977).

6. S. Catto. Exceptional projective geometries and internal symmetries. ArXiv: Hep-th/0302079.

7. G. 't Hooft. A planar diagram theory for strong interactions. Nucl. Phys. B 72, 461 (1974).
8. K. Johnson, C.B. Thorn. Stringlike solutions of the bag model. Phys. Rev. D 13, 1934 (1976).

9. A. Chodos, R.L. Jaffe, K. Johnson, C.B. Thorn. Baryon structure in the bag theory. Phys. Rev. D 10, 2599 (1974).

10. T. De Grand, R.L. Jaffe, K. Johnson, J. Kiskis. Masses and other parameters of the light hadrons. Phys. Rev. D 12, 2060 (1975).

11. S.W. Otto, J.D. Stark. SU(3) heavy-quark potential with high statistics. Phys. Rev. Lett. 52, 2328 (1984).

12. P. Goddard, J. Goldstone, C. Rebbi, C.B. Thorn. Quantum dynamics of a massless relativistic string. Nucl. Phys. B 56, 109 (1973).

13. A. Chodos et al. New extended model of hadrons. Phys. Rev. D 9, 3471 (1974).

14. V.G. Kac. A sketch of Lie superalgebra theory. Comm. Math. Phys. 53, 31 (1977).

Received 08.07.19

С. Катто, І. Гюркан,

Ф. Халъфан, Д. Курт, Б. Ніколеску, Е. Ю

\section{ГАДРОННА СУПЕРСИМЕТРІЯ 3 КХД}

$\mathrm{P}$ е $з$ ю м е

Запропоновано модифікацію масових формул для гадронів, з наголосом на теоретико-груповий опис і суперсиметрію, яка відповідає КХД і базується на кварк-антикварковій симетрії, із подальшими коментарями щодо можливих застосувань до моделей типу Скірма, які в майбутньому можуть конкурувати з потенціальними кварковими моделями. 